\title{
$-O$ \\ Radical Innovation and Network Evolution
}

Sandra Phlippen'

Massimo Riccaboni2

${ }^{\prime}$ Erasmus Universiteit Rotterdam, and Tinbergen Institute;

2 University of Florence, Florence, Italy. 


\section{Tinbergen Institute}

The Tinbergen Institute is the institute for economic research of the Erasmus Universiteit Rotterdam, Universiteit van Amsterdam, and Vrije Universiteit Amsterdam.

Tinbergen Institute Amsterdam

Roetersstraat 31

1018 WB Amsterdam

The Netherlands

Tel.: $\quad+31(0) 205513500$

Fax: $\quad+31(0) 205513555$

Tinbergen Institute Rotterdam

Burg. Oudlaan 50

3062 PA Rotterdam

The Netherlands

Tel.: $\quad+31(0) 104088900$

Fax: $\quad+31(0) 104089031$

Most TI discussion papers can be downloaded at http:/ /www.tinbergen.nl. 


\title{
Radical innovation and network evolution
}

\section{The effect of the genomic revolution on the evolution of the pharmaceutical $R \& D$ network ${ }^{*}$}

\section{Sandra Phlippen ${ }^{* *}$ and Massimo Riccaboni ${ }^{\Upsilon}$}

April 2007

\begin{abstract}
This paper examines how a radical technological innovation affects alliance formation of firms and subsequent network structures. We use longitudinal data of interfirm R\&D collaborations in the biopharmaceutical industry in which a new technological regime is established. Our findings suggest that it requires radical technological change for firms to leave their embedded path of existing alliances and form new alliances with new partners. While new partners are mostly found through the firms' existing network, we provide some insight into distant link formation with unknown partners, which contributes to our understanding of how 'small-worlds' might emerge.
\end{abstract}

\footnotetext{
* I would like to thank participants of the Tinbergen lunch seminars in Rotterdam and Amsterdam for helpful discussions. Especially Michiel de Pooter and Francesco Ravazzolo have given valuable help. Further I would like to thank the participants of the Fourth European Meeting on Applied Evolutionary Economics (EMAEE) in Utrecht and the participants of the Annual Conference ADRES 2006 in Saint -Etienne on Networks of Innovation and Spatial Analysis of Knowledge Diffusion for useful comments. I greatly benefitted from comments and discussion with Marco van der Leij, Robert Dur, Bart Verspagen and Bert van der Knaap. Data stem from the Biotechnology Industry Database, originating from the University of Siena. Financial support was given by the Erasmus Trustfonds in Rotterdam.

** Tinbergen Instituut, Erasmus Universiteit Rotterdam. Address: Burgemeester Oudlaan 50, 3062 PA Rotterdam, The Netherlands. Phone: +31 (0)10 4088924. Fax: (0)10 4089031. Email: phlippen@few.eur.nl

${ }^{\Upsilon}$ Faculty of Economics, University of Florence, Italy. Email: riccaboni@unifi.it
} 


\section{Introduction}

Networks of collaborative relationships among firms are an important form of organization of innovative activities (Powell et al., 1996; Kogut, 2000). Especially in innovative-, and technology intensive industries, firms increasingly realize that, in order to tap into new technologies and know- how, internal development needs to be complemented with strategic collaborations (Gulati, 1998; Verspagen \& Duysters, 2004). In the biopharmaceutical industry, the emergence of an expanding network of R\&D collaborations has been studied intensively (Pisano, 1991; Arora and Gambardella, 1994; Powell et al., 1996; Stuart et al. 1999; Orsenigo et al., 2001; Pammolli et al. 2001; Riccaboni and Pammolli 2002).

In this paper we study the network of innovators in the biopharmaceutical industry to provide a deeper understanding of the underlying mechanisms that drive network structures. We argue that real world networks do not evolve in isolation, but co-evolve along technological paradigms. Our data cover a time frame of 20 years in the biopharmaceutical industry in which a new technological paradigm is established over an old one. The technological transition has been induced by a radical scientific innovation. This has given us the opportunity to explore the effect of radical technological change on structural network evolution.

While radical technological change occurs regularly and has an important influence on the structure of high technology industries and networks (Anderson \& Tushman, 1986), we have found that most inter-firm network studies assume the underlying technology base to be stable. Through this assumption, the majority of these studies assume network evolution to be an endogenous process where network structures guide organizational action and vice versa. Our paper provides additional insights into network evolution, because exogenous influences can lead to relational behavior that cannot be explained from an endogenous perspective on network evolution. Another, more theoretical contribution of our paper relates to the notion of small worlds in complex networks. While most recent studies on small-world networks reveal the existence and topology of such a network structure, this paper is, as far as we know, the first study to look at influences that might induce the relational behavior that leads to a smallworld structure.

We find that the genomic revolution, representing an exogenous shock, leads to an expansion of the network through a wave of firm entry and a wave of alliance formation. When looking more specifically at the partners of new alliances, we find that it requires an exogenous shock like the genomic revolution, for firms to leave their embedded path of existing collaborations and ally with new partners. For managers of new firms or peripheral firms, knowledge about 
such network changes creates an opportunity to potentially improve one's position in the network. Especially in industries characterized by hierarchical structures and low turnover, where large incumbent firms are dominant and newcomers are usually specialized niche players, knowledge about such structural breakthroughs can be crucial. While it is interesting for firms to be able to anticipate the effect of radical innovation on their and their competitors' position in a network, policy makers can also benefit from this knowledge. Since radical scientific innovation is often induced by government-led R\&D programs, policy makers should know about the potential effect of publicly financed R\&D projects on firm behavior and network development.

In what follows we first elaborate rather extensively on the existing literature, because our aim is to connect insights from organization-, and strategic management literature to issues in complex network theory. At the intersection of these two strands of literature we find relational behavior that can only be explained when taking exogenous influences into account. In section 2, we introduce our measurement techniques for measuring structural network change, such as the clustering coefficient and the average path-length. Section 3 focuses on exogenous influences on the network. In this section we introduce the reader to our empirical setting, the genomic revolution. We apply and extend a theoretical framework developed by Koka et al. (2006), which enables us to hypothesize on the effect that the genomic revolution has had on the pharmaceutical R\&D network in section 4. We divide this section into hypothesized structural change at the level of the network, and into structural change at the level of the firm, whereby this latter part touches upon specific relational behavior that is associated with small worlds. After describing the data and methodology in section 5, we present the results in section 6 and a discussion and conclusion in section 7 and 8 respectively.

\section{Network evolution}

The understanding of how networks evolve has been a topic of interest to both social scientists and natural scientists. Both scholars realize that relational behavior and network structure are intertwined. An actors' relational strategy depends for some part on the structure of relationships it had before. At the same time, the actors' new relationships contribute to a changed network structure that again influences its actions (Gulati, 1998). Social scientists and natural scientists differ in regard to the way they study network evolution. Social scientists assume that actors conduct strategic relational behavior, while natural scientists, studying complex network theory, often assume actors to be non-human (e.g. proteins). 
Amongst the latter, the process of link formation is based on certain 'rules of attachment'. Recently, a number of authors such as Goyal et al.(2006), Uzi \& Spiro (2005), Wilwhite (2001), Verspagen \& Duysters (2004), and Jackson (2006) have combined insights from both strands of literature and increased our understanding of network evolution. Our paper aims to contribute to this understanding.

Network change consists of changes in the number of actors (exit and entry), and changes in numbers-, and patterns of link formation (Koka, 2006). Structural network change is a form of network change whereby new linkages are formed with new partners. Studies on new partner search in networks have broadly focused on two issues. One issue is about distribution of linkages among actors in a network, which represents the inequality of access that firms have to various resources. In many real world networks the distribution of linkages among actors is highly unequal (Dorogovtsev and Mendez, 2003; Goyal, et al.2005; Barabasi et al., 2002). Barabasi (1998) shows how actors accumulate new linkages in proportion to the number of linkages they have already (preferential attachment). Following from this 'rich get richer' principle of growth, the resulting network structure consists of a few highly connected actors called 'stars' in combination and many weakly connected 'peripheral' players. The second issue in new partner search concerns the process of local link formation and the process of distant link formation, which will be the focus of this study.

\section{Local link formation}

Local link formation implies that new partners are found through an actors' existing network (which is called an ego network), and that the new partner is already known to other partners 'in the neighborhood'. The overall network structure resulting from local link formation is a network composed of dense 'cliques' of actors, which indicates that they are highly connected to each other. Local link formation of an actor and the degree of clique formation in a network can be measured by calculating the clustering coefficient, which will be explained in section 2 .

Within the organization-, and strategic management literature, network studies mainly focus on the effect that a given network structure has on the relational behavior and performance of firms (Burt, 1992; Granovetter, 1985; Gulati et al., 2000). Having a more central and autonomous structural position in a network provides firms with access to resources, learning opportunities, and reduces uncertainty (A notion first coined by sociologists like Bourdieu (1980) and Coleman (1990) as 'social capital'). Regarding the formation of new linkages, Gulati (1995) finds that the process of new tie creation is heavily embedded in an actors 
existing network (consisting of previous alliances). This means that new ties are often formed with prior partners or with partners of prior partners (Gulati, 1995), indicating network growth to be a 'local' process, where strategic collaborations are path-dependant (Noria, 1992). Particularly when considering inter-firm alliances, new link formation is considered 'risky business' and actors prefer alliances that are embedded in a dense clique were norms are more likely to be enforceable and opportunistic behavior to be punished (Gulati 1995; Powell et al., 1996; Koka et al., 2006; Granovetter, 1985).

\section{Distant link formation}

Distant link formation implies that new linkages are created with partners whom are not known to the existing partners of an actor. In the social sciences, Granovetter (1985) was the first to differentiate between local ties in dense cliques (strong ties) and distant ties that bridge these cliques (weak ties). More precisely, the author argues that distant linkages "serve as crucial functions in linking otherwise unconnected segments of the network" (Granovetter, 1983: 217). At the level of the firm, Burt (1992) shows that distant linkages that serve as bridges between dense local cliques of firms, can provide access to new sources of information and favorable strategic negotiating positions (termed 'structural holes'), which improves the firms' position in the network and industry.

\section{Small worlds}

The first network studies that combine local- and distant link formation originate from complex network studies. Watts and Strogatz (1998) model the process of local link formation and find that, with the addition of just a handful of distant linkages, a specific network structure is generated, which they call a small world. This means that although large networks have relatively few linkages compared to the number of actors, the reach is higher than expected (Newman, 2001). While solely local link formation results in dense cliques of connected actors, the average distance to reach all actors in a network is very large. The distance between two actors is indicated by the number of other actors one has to surpass in order to reach the other. Watts \& Strogatz (1998) found that the average distance between all actors in a network is sharply reduced when a relatively small number of distant linkages (referred to as random linkages) are added to the network that serve as shortcuts between these local cliques. Examples of small world networks are the electronic power grid network, high-school friendship networks, or the neural network of a worm (see for an overview Watts (1999) or Newman (2001)). Recently, insights from the social sciences regarding network 
evolution and new link formation have been combined with the more theoretical findings as described above (see Goyal, et al., 2006; Uzi \& Spiro, 2005; Wilwhite, 2001; Verspagen \& Duysters, 2004). Verspagen \& Duysters (2004) explain how firms that try to build 'social capital' can be seen as drivers of local link formation, and firms that strategically aim to bridge structural holes in a network can be seen as drivers of distant link formation. Together, these two drivers of new partner search add up to small world structures in networks of technology alliances. A recent study by Jackson \& Rogers (2006) focuses on link formation with new partners in social networks. They find that large social networks evolve into small worlds, because people meet friends of friends and strangers. The process of link formation is generated by an algorithm that makes actors form both local linkages and random linkages (distant linkages), while implying that random link formation resembles the "meeting of strangers'. While the latter implication seems feasible in friendship networks, random partner search by firms seems rather unlikely. A plausible assumption made by Verspagen \& Duysters (2004), is that firms are aware of the structural features of the network surrounding them and that this induces these firms to deliberately form distant linkages that bridge local cliques. However, a number of studies such as the work of Cowan et al. (2004) and Powell (1990) emphasize the risks involved in new partner formation, and the strong tendency of these firms to use their existing network as a source of information for new partner search, implying local link formation.

In this paper we aim to contribute to the understanding of local-, and distant link formation in inter-firm alliance networks. Moreover, we investigate the effect of a radical exogenous innovation on structural network change. This paper differs from the studies mentioned above, in that these studies analyze the effect of link formation on the emergence of a smallworld. Our study investigates whether radical exogenous change induces link formation, which potentially leads to a small world. We use the theoretical framework of Koka et al. (2006) to measure structural change, and we will expand their framework by introducing local-, and distant link formation.

\section{Structural change}

Koka et al.(2006) have combined multiple indicators of relational behavior into four different types of network change (see figure 1). The network can expand, churn, strengthen or shrink. Each network change is brought about by a specific combination of changes in tie creation, tie deletion, and by changes in an actor's portfolio size (number of links) and portfolio range (number of partners). 
Fig.1- Environmental effects on patterns of network change

\begin{tabular}{|c|c|c|}
\hline & Changes in unc & \\
\hline & Increase & Decrease \\
\hline & Network expansion & Network strengthening \\
\hline Increase & Tie creation: Increase & Tie creation: Increase \\
\hline & Tie deletion: Decrease & Tie deletion: Decrease \\
\hline & Portfolio size: Increase & Portfolio size: Increase \\
\hline & Portfolio range: Increase & Portfolio range: Decrease \\
\hline Changes in Munificence & & \\
\hline & Network churning & Network shrinking \\
\hline Decrease & Tie creation: Increase & Tie creation: Decrease \\
\hline & Tie deletion: Increase & Tie deletion: Increase \\
\hline & Portfolio size: Little change & Portfolio size: Decrease \\
\hline & Portfolio range: Increase & Portfolio range: Decrease \\
\hline
\end{tabular}

Source: Koka et al., 2006

While Koka et al.(2006) present four types of network change they find that only an expanding network and a churning network are a reflection of structural change, because new alliances are formed with new partners. An expanding network is brought about by an increase in new alliances without deletion of old alliances (meaning a larger average portfolio), together with an increasing portfolio range (more different partners). A churning network reflects the formation of new alliances and the deletion of existing alliances. While the average portfolio remains stable in terms of the number of partners, there is an increasing variety in identity of partners. We will use this framework to hypothesize on the 'type' of network change to expect after a given exogenous or environmental change. While changes in the number of linkages (tie creation/deletion) and changes in the number and identity of partners already provides important insights into structural changes in the network, we will further distinguish between local link formation and distant link formation when studying new link formation with new partners. Local link formation and distant link formation are 
measured through the calculation of the clustering coefficient and the average distance between actors respectively.

\section{Clustering coefficient}

The clustering coefficient of an actor is the density of its open neighborhood, that is to say how close each actor's neighborhood is to a fully connected clique. Following Watts and Strogatz (1998), we define a clustering coefficient as follows: assume that the $i$ th vertex $v_{i}$ has $k_{i}-1$ neighboring vertices. At most, $k_{i}\left(k_{i}-1\right) / 2$ edges can exist between them. Calculate $c_{i} \equiv$ (number of edges of $v_{i}$ and its neighbors) $/ k_{i}\left(k_{i}-1\right) / 2$. Then, the overall clustering coefficient is defined by $C C=\frac{1}{N} \sum_{i=1}^{N} c_{i}$, which is the average of the individual clustering coefficients $c_{i}$. The weighted overall clustering coefficient (WCC) is the weighted mean of the clustering coefficient of all the actors each one weighted by its degree. This last figure is exactly the same as the transitivity index of each transitive triple expressed as a percentage of the triples in which there is a path from $i$ to $j^{l}$. The cluster coefficient tends to 1 if most of the partners of each biopharmaceutical institution are directly related by formal $R \& D$ collaborations. On the contrary, the clustering coefficient tends to 0 if the network is hierarchical and the partners of each biopharmaceutical actor are not related.

Clustering coefficients are often applied to detect small-world networks and the degree of hierarchy of local relational structures. At the level of the network, the degree of hierarchy of local relational structures is called transitivity. If an actor's ego-network is a clique, meaning the absence of hierarchy, the actor and its partners all have equal structural power and the network as a whole becomes more transitive.

Because the clustering coefficient (CC) essentially measures the situation whether an actor's partners are connected to each other, an increasing CC measures new alliances being created with partners that are already known to the partners in the clique. We can visualize this alliance behavior as follows:

\footnotetext{
${ }^{1}$ To calculate $\mathrm{C}$ it is important to notice that there are no loops attached to a vertex (no self-ties) and that multiple relationships between two vertices are identified as one edge.
} 
Fig. 2 - Visualization of local link formation
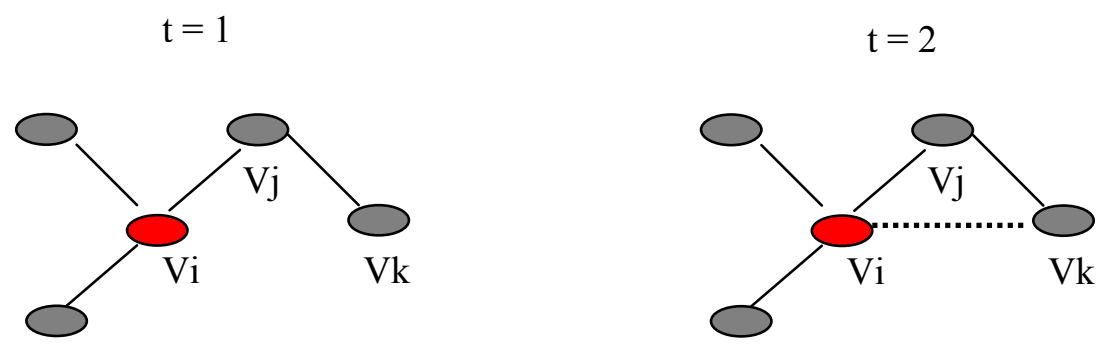

In figure 2 we can easily see that whereas at $\mathrm{t}=1$ vertex $i$ 's partners are unconnected, the alliance between vertex $i$ and vertex $k$ at time 2 indicates that $i$ has a new partner who already was a partner of $j$. The clustering coefficient has risen from $0(0 / 3)$ to $0.167(1 / 6)$. To account for the size of a firms' ego network, we will use changes in the weighted clustering coefficient as an indicator of structural network change, because it reflects the formation of new alliances with new 'local' partners whom are already known to the actors in a clique.

\section{Average path-length}

To measure structural network change that is caused by new link generation between local clusters, we use the average path-length in the network. Following Goyal et al.(2006), we define the average path-length between reachable pairs in our network as $d(N)$, being the average distance between any actor $i$ and $j$ that belong to the same component. Actors that are isolated from other actors are excluded. Thus, for a connected graph $N(E, V)$ consisting of edges and vertices, the average path-length is the sum of the distances between two actors $(i$ and $j$ ) belonging to the network $(N)$, divided by all possible edges excluding self-ties:

$$
d(N)=\frac{\sum_{j \in V} \sum_{i \in V} d(i, j ; N)}{v(v-1)}
$$

The above definition of average path-length is only useful when most actors in a network under study do indeed belong to the same component. To verify this, we plotted the number of actors within the main component of our network in relation to the all actors in the network 
in figure 3. Clearly, this figure shows that at any moment in time, most actors belong to the main component in which every actor is connected.

\section{Fig.3 - Size of the main component (dotted line) in relation to the whole network (full line)}

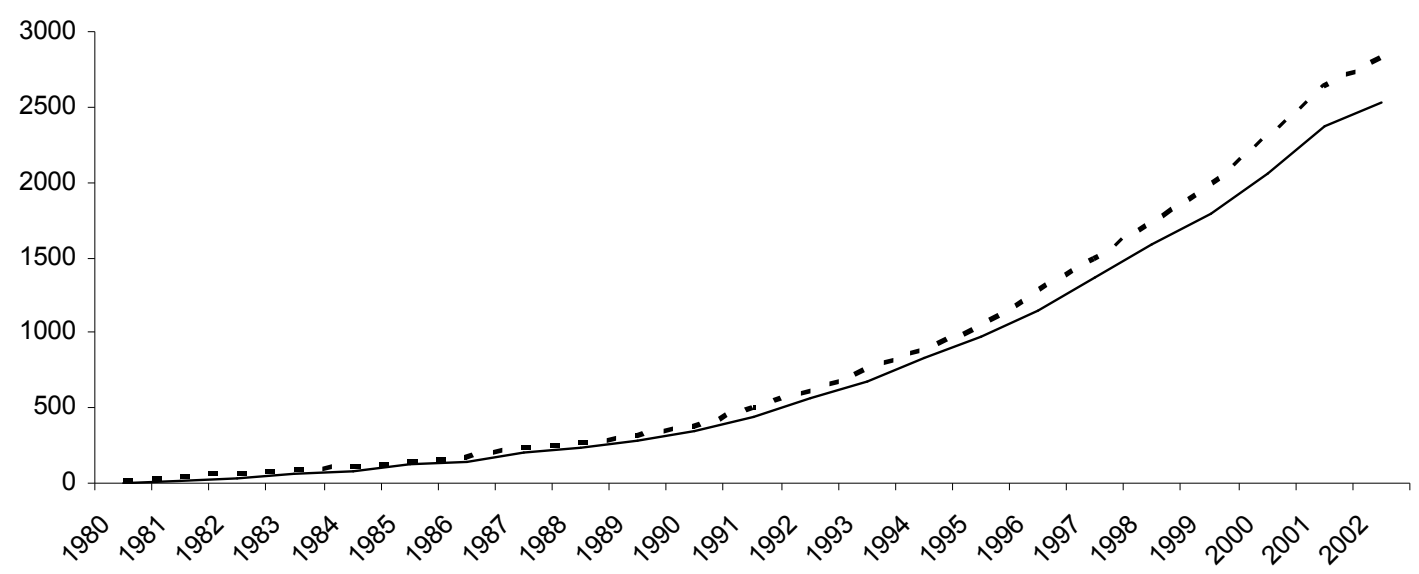

While average path-length is an indicator of the overall network structure, the change in average path-length over time provides information of structural network change at the intermediate level. Moreover, in social networks where the majority of link formation occurs within the neighborhood, only the occurrence of a relatively few number of distant linkages between neighborhoods can cause the average path-length in a network to fall. This is because a connection (bridge) between to isolated clusters of actors suddenly increases the reach between those clusters and thus decreases the path-length. The above described argument originates from Watts and Strogatz (1998) in their seminal paper on small-worlds. For our paper we turn this argument around by assuming that a decreasing average path-length is a structural indicator that some actors in our network have been able to form distant linkages with new partners who are not familiar to the actors existing partners. The average pathlength is thus an indirect measure of distant linkages, and one could argue that it would be better to measure distant linkages directly. We argue however, that the usage of average pathlength has the great advantage of only measuring 'distant linkages' that are effective in providing positional benefits to the firms involved. In order to explain why, we have visualized the ego networks of two vertices (hypothetical firms) $\mathrm{V} i$ and $\mathrm{V} j$ as follows: 
Fig. 4 - Visualization of (non effective) distant link formation

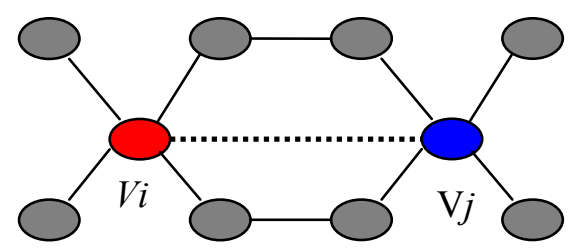

If we were to measure distant link formation 'directly', we would say that a link between two firms is a distant link, if the ego networks of both partners are non-overlapping. When using this definition the link between $i$ and $j$ in figure 4 can be defined as a distant link. However, we can also see that both firms are part of the same clique because their partners' partners are highly connected. This would make the 'distant link' between $i$ and $j$ much less valuable in terms of improvement of their structural network position or in terms of spreading information through the net. In figure 4, the dotted line between $i$ and $j$ represents such a 'less informative' distant link. While $i$ and $j$ do not share partners, their link is not likely to bring new information to the group.

At the level of the network, the average path-length only decreases when distant linkages are formed that provide real shortcuts in the network, meaning that they connect cliques that where unconnected before. Linkages (dotted) that connect parts of a network in a way which reduces the average distance have been visualized in figure $5^{2}$.

\footnotetext{
${ }^{2}$ This visualization is merely intended to clarify the difference between two ways of measuring distant link formation. The Authors are aware that this visualization cannot occur empirically because only connected actors are included in graph $N(E, V)$.
} 
Fig. 5 - Visualization of effective distant link formation (dotted lines)

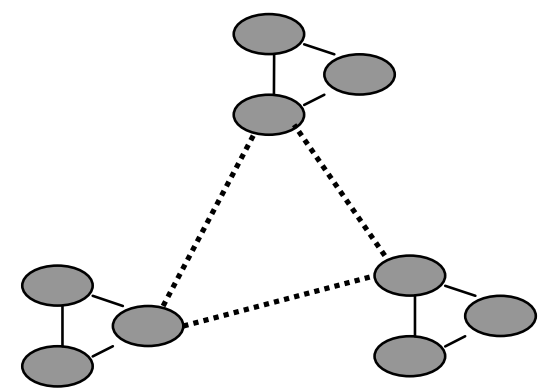

In order to understand why actors are sometimes able to create these distant linkages, and thus shorten the average distance in the network, we explore the influence of exogenous forces on the evolution of the network.

\section{Exogenous influences on structural network change}

Various studies focusing on structural network change have argued that real structural change only occurs after an exogenous shock (Barley, 1986; Piore \& Sabel, 1984; Glasmeier, 1991). There are few networks studies in the organization- and management literature which take exogenous influences on network evolution into account. One important contribution comes from Madhavan, Koka, and Prescott (1998) with their study on the effect of a technological and regulatory 'event' on structural network change. Madhaven et al. (1998) find that it requires a radical technological change to enable relatively peripheral players in the network to significantly improve their network position and consequently cause a 'loosened' network structure. Koka et al. (2006) have expanded and generalized their work by providing a framework to assess the effects of exogenous events on structural network change (figure 1).

Exogenous or environmental changes are expressed in terms of changes in uncertainty and munificence instead of specific industry events, which makes the framework a useful metatool for broader applications. Following Dickson \& Weaver (1997) the authors define uncertainty as "the inability of a firm's managers to accurately assess the external environment of the organization or the future changes that might occur in that environment". Uncertainty induces alliance activity (Nohria \& Garcia-Pont, 1991; Powell, et al., 1996). But, whereas some authors argue that new alliances are used to reinforce a firm's relationships with existing partners during uncertainty (see Granovetter (1982) and Krackhardt (1992)), others, such as Kogut (1991), find that firms might create new alliances with new partners in 
order to expand its number of strategic options as a means to cope with uncertainty. To resolve the issue of either reinforcement of existing relations or the formation of new relations with new partners, the authors introduce to concept of munificence. Munificence refers to the "extend to which resources available to a firm are plentiful or scarce, after taking into account the number of firms competing for those resources" (Koka et al.2006:725). While uncertainty increases the array of actions firms can potentially make in the changing environment, the opportunities to do so are limited by the resources available to the firm. In short, we can say that while uncertainty represents the opportunity for alliance formation with new firms, munificence represents the ability for alliance formation with new firms, given the opportunity to do so.

We use the concepts of uncertainty and munificence to hypothesize on how the genomic revolution in the beginning of the nineties has induced structural change in the pharmaceutical R\&D network. In the next section, we will analyze the genomic revolution in terms of its influence on uncertainty and munificence. From there we derive our hypothesis on how we expect genomic revolution to induce relational behavior that causes structural network change.

\section{The genomic revolution}

The genomic revolution represents the radical scientific innovations related to the identification and understanding of the human genome and the technologies to store and analyze genetic information ${ }^{3}$.

Our aim is to provide insight in how radical technological change such as the genomic revolution has caused structural network change. Before getting to the question of 'how', we need to defend the causal order of the question. The relational behavior of network actors could after all have created the genomic revolution and not vice versa. In order to disentangle the genomic revolution from the changes in the pharmaceutical R\&D network, we show that the genomic revolution was sparked by a government led R\&D program that had started long before structural changes in the network became apparent. Second, in order to be sure that government-led or government-financed R\&D is not driving the structural network change,

\footnotetext{
${ }^{3}$ See the website of the U.S. Department of Energy Office of Science for information on the genomic revolution and the Human Genome Project. (http://www.ornl.gov/sci/techresources/Human_Genome/home.shtml)
} 
we excluded government and academic actors from the R\&D network we study, thereby focusing on the industrial $R \& D$ network.

\section{Human Genome project}

The Human Genome Project is a government led research project to unravel the human genetic code. The very first initiatives for the project were undertaken in 1983 by the US energy department laboratory, with the creation of DNA clone libraries representing single chromosomes. At least until 1988, the only active institutions that were involved in the setup of the human genome project were the US department of energy and the National Institute of Health. The main aims of the project were the identification of all genes and determination of sequences of chemical base pairs in human DNA, the development of storage capacity and analysis tools of genetic information, and the transference of related technologies to the private sector. The project has been completed in $2003^{4}$.

\section{General purpose technologies}

While the Human Genome project had brought forward huge amounts of new information on genetic targets, new tools for drug discovery were needed to deal with the available genetic information. These new drug discovery tools such as combinatorial chemistry, high throughput screening and bioinformatics are not only different from conventional medicinal chemistry because they enable the testing of larger amounts of chemical entities against more drug targets, they are also much more broadly applicable in terms of disease areas and biological targets (Orsenigo, et al., 2001). Based on these new tools known as general purpose technologies (GPT), a wave of new firms specializing in GPT had been founded. In figure 6 we show how the proportion of alliances based on general purpose technologies has overtaken the proportion of alliances based on conventional medicinal chemistry (co-specialized technologies) in the beginning of the nineties.

\footnotetext{
${ }^{4}$ See for the timeline of developments of the Human Genome project: www.http://doegenomes.org/
} 
Fig. 6 - Proportion of alliances based on General Purpose Technologies (genomics, proteomics, bioinformatics, combinatorial chemistry, high-throughput screening)

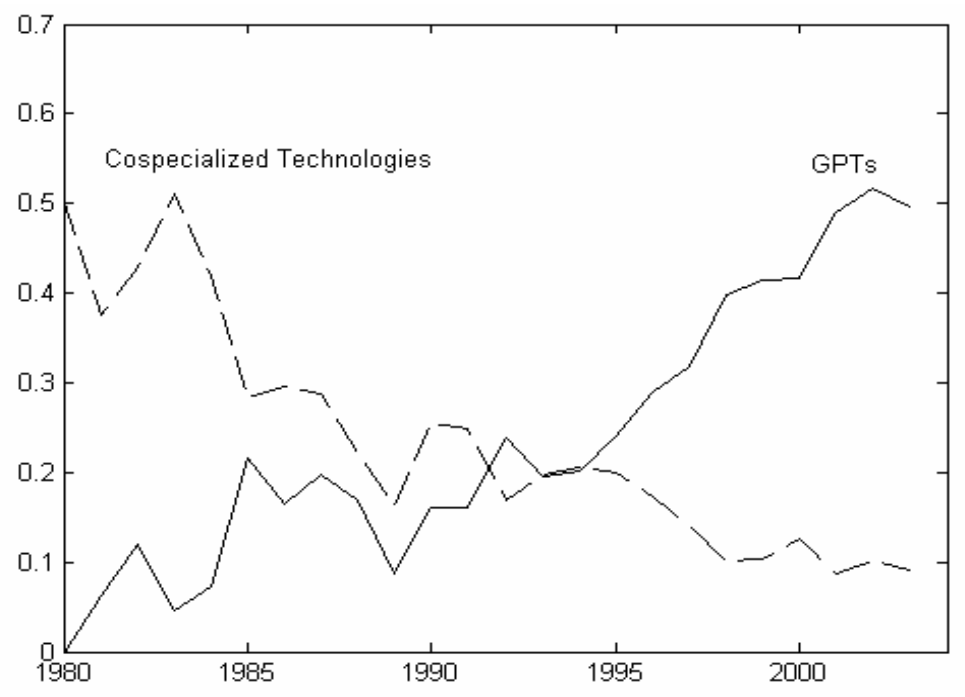

The activities of general purpose technology based firms (GPT based firms) differ from other firms' activities in drug development because they provide tools for drug development instead of developing a specific drug (Kaplan et al., 2003). According to business analysts, such as Longman (2000) and Lytton (1999), the different relational behavior of GPT firms compared to 'traditional' (co-specialized) firms, can be partly attributed to the specific characteristics of general purpose based technologies.

\section{Effects of the radical technological change}

In this section of the paper we first investigate the effect that the genomic revolution has had on changes in munificence and uncertainty. Through these changes we hypothesize on the effects of the genomic revolution on structural network change. In the second part of the section we expand the concept of structural change by distinguishing new alliances with new 'local' partners from new alliances with new 'distant' partners. Through this approach our findings contribute to the literature on small-world networks and to the notion of the factors driving a small-world.

\section{Structural change: uncertainty and munificence after the genomic revolution}

The genomic revolution encompasses a number of radical scientific and technological innovations that have and are altering existing practices of drug development (Uppenbrink \& 
Mervis, 2000; Gassmann et al.2004). While traditional approaches of medicinal chemistry and sequential experimentation have by no means become redundant, they have been complemented and intensified by general purpose technologies of which high-throughput screening, combinatorial chemistry, bio-informatics, proteomics, genomics, pharmacogenomics, and molecular design are the most important. Although these technologies are very heterogeneous in their function, together they are responsible for the alteration of drug development into a more automated, mass production process based on trial and error (Gassmann et al.2004; Nightingale, 2000; Drews, 2000). GPT are poised to improve the process of drug discovery in revolutionary ways, but there are also concerns about the increased complexity and diversity that these technologies bring to the drug development process (Longman, 2000; Orsenigo et al., 2001; Drews, 2000). Burckhardt \& Brass (1990) and Hannan \& Freeman (1989) find that technological change creates uncertainty, because of increased heterogeneity and complexity. Following this line of reasoning, we assume that the genomic revolution has increased environmental uncertainty.

Munificence has also increased after the genomics revolution ${ }^{5}$. Munificence reflects a firms' capacity (Dess \& Beard, 1984). By using various new tools, general purpose technologies have greatly increased the number of possible strategies for drug discovery. This can be considered as an increase in technological resources. Adding to the notion of increasing munificence is the favorable investment climate after the Human Genome Project proved successful. Mainly general purpose based firms benefited from the willingness of investors to put their money in start-up companies that take no risks in drug development itself, but only provide the tools (Longman, 2001). The availability of technological resources together with the financial capacity to develop or invest in these resources, accounts for an increase in munificence. The increase in munificence is somewhat moderated by the wave of entrance of general purpose based firms which increases competition and lowers the average increase in munificence per firm. However, we consider firm entry to be a consequence of increasing munificence rather that a potential cause of reduced munificence. Concluding, we assume that

\footnotetext{
${ }^{5}$ The authors realize that while munificence clearly increased in the beginning of the nineties with the general believe that genomic based technologies would revolutionize drug development, later there has been some doubt about the revolutionizing effect of genomics. So far, drug development has mainly become more complex through all these new alternatives for development (Drews, 2000). Some analysts even say that the genomics revolution has decreased efficiency of drug development because of the decreasing number of scientists working together on one disease target. The increase in genetic targets has caused spreading of researchers over the different targets which slows down the discovery process (Longman, 2000).
} 
both uncertainty and munificence have increased after the genomic revolution in the beginning of the nineties. Following the framework of Koka et al. (2006; see figure 1), we arrive at the following hypothesis on the effect that the genomic revolution has had on the structural changes in the pharmaceutical R\&D network.

\section{Hypothesis 1}

Because the genomic revolution has increased both environmental uncertainty and munificence, we expect to see an expansion of the pharmaceutical R\&D network, which we measure through a higher average portfolio size of alliances per firm in combination with an increased average range of partners per firm.

\section{Structural change: local clustering and distant linkages}

In order to be able to hypothesize on which type of relational behavior (local link formation and/or distant link formation) to expect of firms after the genomic revolution, we first need to explain the relational behavior of firms before the genomics revolution.

Before the genomic revolution, drug development has been based on molecular biology, biochemistry, pharmacology and other disciplines for many years. Orsenigo et al.(2001) have established a connection between the nature of knowledge advancement in these years and the inter-organizational network structure in the pharmaceutical industry. They found that parallel to research in drug discovery, which develops as a branching process of older more general research hypothesis toward more specialized sub-hypothesis, a similar hierarchal branching structure unfolded in the collaborative $R \& D$ network between organizations. More specifically, the authors find that large, incumbent pharmaceutical firms manage more general knowledge in the network while new entrants (mainly dedicated biotechnology firms) specialize in specific sub-hypothesis of drug research in specialized disease areas and collaborate with the incumbent players. Over time this network evolves into a hierarchal R\&D network consisting of fairly 'isolated' branches which represent specialized fields or disease areas.

After the genomic revolution, firms entered the network through unusual relational behavior. The GPT that these entering firms relied on, do not obey to the 'traditional' logic of knowledge advancement, because they contribute tools to the drug development process instead of developing drugs. With the purpose of being an aid to drug development, GPT are more broadly applied in terms of number of disease areas and biological targets. As a result, 
these firms mostly form non-exclusive alliances with a large variety of firms and are by definition not bound to any specific research field or disease area (Longman, 2000; Lytton, 1999). According to Orsenigo et al. (2001) GPT based firms "pertubate the structure of the network" (Orsenigo et al., 2001:490). Given the relational behavior of existing firms in the pharmaceutical R\&D network in combination with the relational behavior of general purpose based firms entering the network after the genomic revolution, we expect the network structure to be affected in the following way:

Fig. 7 - Simplified network topologies

Before the genomic revolution

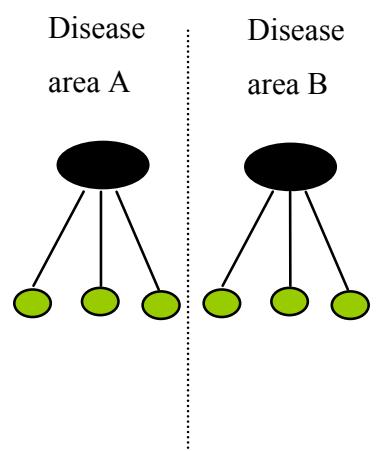

General purpose based firms

Specialized biotech firms

Large incumbent pharmaceutical
After the genomic revolution
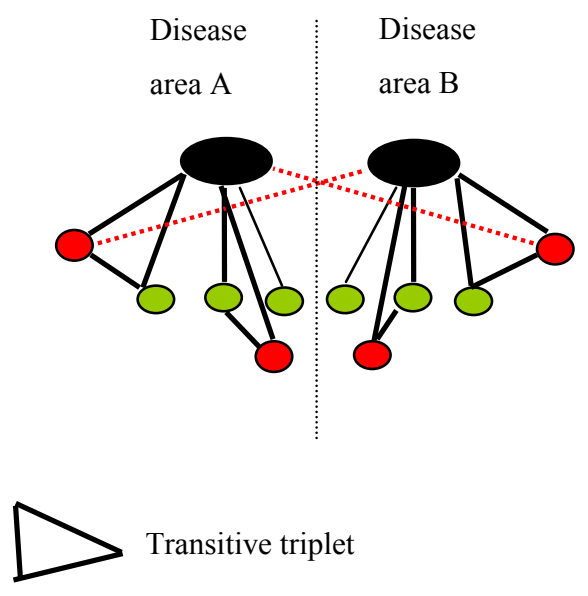

'Distant linkage'

The first picture in figure 7 represents a simplified topology of the pharmaceutical R\&D network before the genomic revolution. Within each branch (disease area A and B), specialized biotech firms collaborate with large pharmaceutical firms. It is clear that the network is organized in a hierarchal manner within isolated branches of disease areas. While this is obviously an under-representation of the complexity of the real pharmaceutical network structure, it gives us the opportunity to envision what happens when general purpose based firms enter the network with different relational behavior. The second picture in figure 7 shows the entrance of general purpose based firms (red nodes). The linkages they form are based on the notion that they form non-exclusive alliances with a large variety of firms and that they are by definition not bound to any specific research field or disease area. Every linkage formed by newly entering general purpose based firms, is a new linkage with a new 
partner, and these linkages thus cause structural change according to our definition of structural network change. The results from the new link formation of general purpose based firms become apparent when we look at the black triangles and red (dotted) lines. The black triangles indicate that new alliances are formed locally and result in dense cliques. The red lines indicate distant linkages because they connect to distant partners from different disease areas. We can now formulate our hypothesis as follows:

\section{Hypothesis 2 a}

The genomic revolution has induced new partner search through local link formation. This relational behavior results in an increased clustering coefficient in the pharmaceutical $R \& D$ network from the beginning of the nineties.

\section{Hypothesis $2 \mathrm{~b}$}

The genomic revolution has led to firms forming distant linkages between disease areas which are otherwise relatively unconnected. This relational behavior results in a decreasing average path-length of the pharmaceutical R\&D network from the beginning of the nineties.

Summarizing our analytical approach in figure 8 , we can say that exogenous change induces relational behavior that is reflected in a changed network structure. More specifically, we argue that the genomic revolution is a radical scientific innovation that is exogenous to our network. This innovation has increased environmental uncertainty and munificence for firms, who respond by increasing their portfolio size and range, leading to network expansion. In more detail, we expect that GPT based firms connect both local players within a disease area into more dense cliques (clustering coefficient), and that they connect these cliques through 'distant linkages' between disease areas (average path-length). 
Fig. 8 - Analytical approach

\begin{tabular}{c|c|c} 
(radical) Exogenous change & Relational behavior & Structural change \\
\hline $\begin{array}{c}\text { Genomic revolution: } \\
\text { general purpose } \\
\text { technologies }\end{array}$ & $\begin{array}{c}\text { Portfolio size }+ \\
\text { Portfolio range }+\end{array}$ & Network expansion \\
Uncertainty + & Local link formation + & Clustering coëfficient + \\
Munificence + & Weak link formation + & Average pathlength - \\
& &
\end{tabular}

\section{Data and methods}

For our empirical analysis we have used a comprehensive and original data set that encompasses information about collaborative agreements in the biopharmaceutical industry worldwide. As a whole, the Biotech Industry Database (referred as BID) covers 20,182 collaborations subscribed by 7,407 institutions including dedicated biotech companies, established companies, specialized biotech suppliers and non-industrial research organizations since 1976. The BID has been created at the University of Siena, and was previously used by Orsenigo, Pammolli, and Riccaboni (2001) to analyze the biopharmaceutical network. As our paper focuses on the pharmaceutical R\&D network our sample consists of 10.580 collaborations among 3800 agents. For each transaction, BID includes information about:

Date of signing (1976-2002);

Partners (classified according to their role in the collaboration);

Stage of development at signing (i.e. discovery, preclinical, clinical...);

Technological content (i.e. gene therapy, genomics, combinatorial chemistry...);

Therapeutic category (i.e. Oncology, Metabolic disorders, Central Nervous System...);

Typology (viz. license, joint venture, co-development...);

Deal value and terms of payment (equity, upfront, milestones, royalties...).

The structure of the network of R\&D collaborations can be represented by a graph $N(E, V)$, where $V$ is the set of vertices (firms), and $E$ are edges (R\&D collaborations). Every edge $e$ within the graph (industry network) is defined as a link between two partners. The graph $N$ can also be represented by an adjacency matrix $N \Leftrightarrow A(N)=[a e]$. Matrix entry ae equals 1 if and only if an edge e does exist, and 0 otherwise. In order to analyze the evolution of our network over time, we took 'snapshots' of the network by labeling each connection with the 
date of signing. The overall graph $N(E, V)$ is decomposed in time specific sub-graphs $N_{\tau}(E, V)$, which include all collaborations up to period $t$.

The first part of the analysis is based on simple count statistics, which reveal the changes in firm entry and alliance formation over time, to see whether the R\&D network has expanded as our first hypothesis predicts. To test hypotheses $2 \mathrm{a}$ and $2 \mathrm{~b}$ we calculate the clustering coefficients and average path-length respectively over time using Ucinet (Borgatti et al., 1999). Based on the adjacency matrix for each time $\operatorname{period} \tau$, we calculate the clustering coefficient and the average path-length. In order to facilitate interpretation of the results we calculate the clustering coefficients and average path-lengths of a random network with the same number of nodes $(V)$ and the same number of linkages $(E)$ at each time period $\tau$.

\section{Results}

\section{Structural change}

Figure 9 shows the number of deal-active firms entering the network $(V)$ and the number of new alliances $(E)$ in the R\&D network from 1967 until 2002. Deal-active firms are firms, which close at least one new alliance in period $\tau$. Until 1992, the number of new alliances formed has been twice as high as the amount of deal-active firms entering the network in each period. After 1992 the grey line in our plot indicates that the amount of new alliances has more or less tripled the number of new deal-active firms. In other words, the average number of new alliances per firm has increased from two to three, which means that the average size of a firms' portfolio of alliances has increased starting in the beginning of the nineties. 
Fig. 9 - Number of new R\&D collaborative agreements (E - Edges) and institutions (V - Vertices) in the network of R\&D collaborations in biopharmaceuticals (19762002)

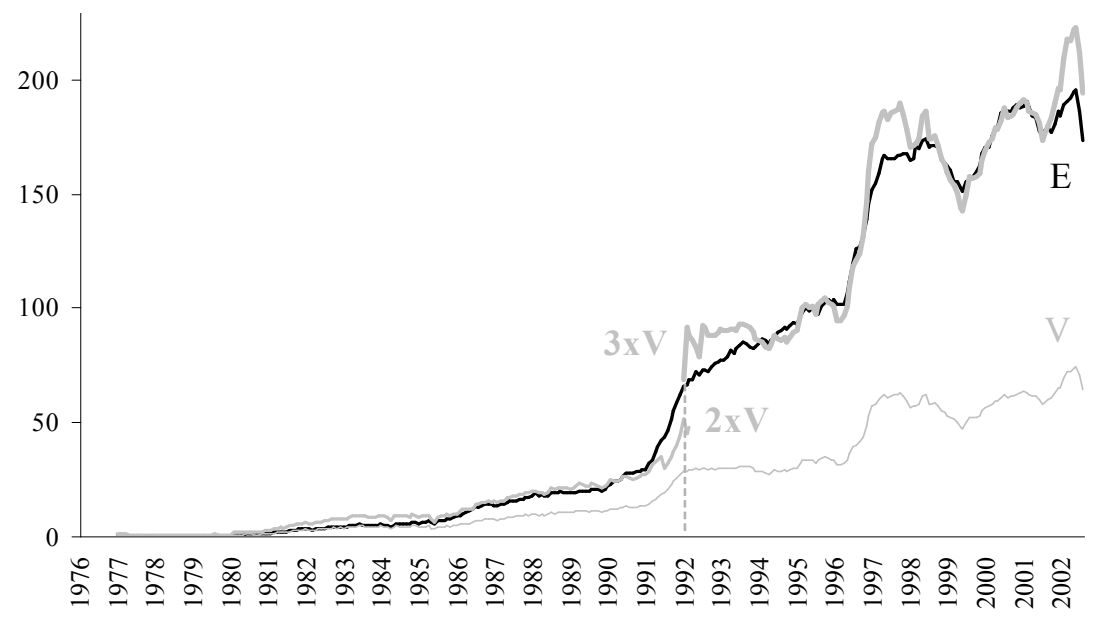

In figure 10 we have plotted the new alliances and new firms in the network in relation to the existing actors and their alliances. We can clearly see that there are various waves of firm entry and alliance growth over time, and the beginning of the nineties marks the start of a new wave of entry and collaboration activity.

Fig. 10 - Percentual change in R\&D collaborative agreements (E - Edges) and institutions (V - Vertices) in the bionharmaceutical R\&D network.

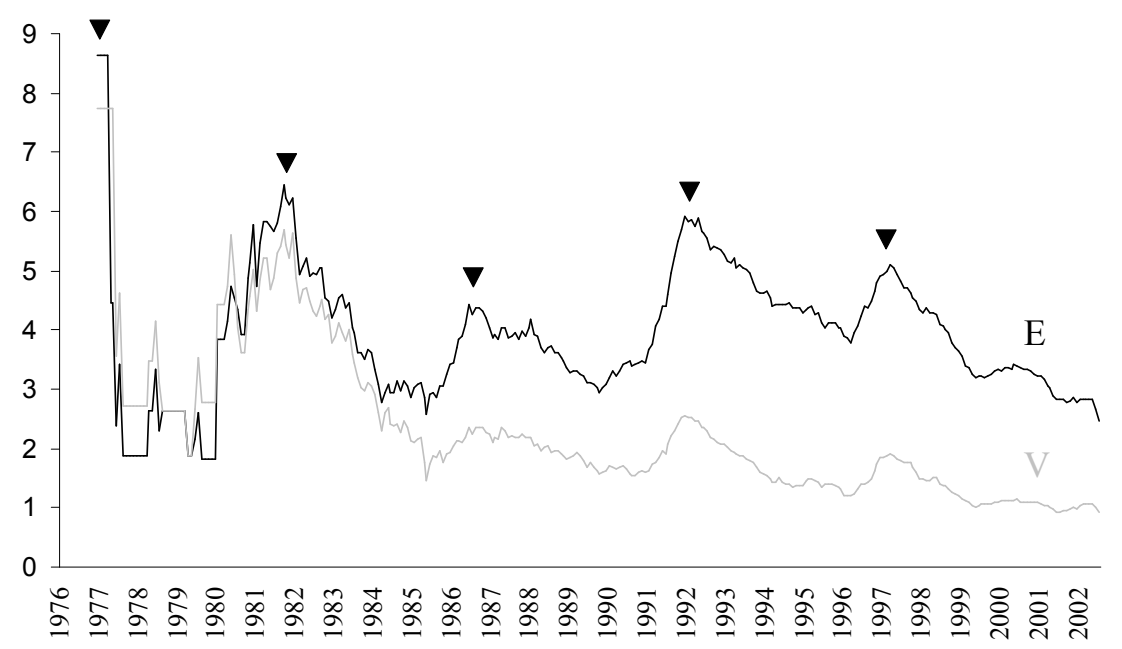

Unless newly entering firms form alliances exclusively amongst each other, a situation which is highly unlikely considering the complementary nature of their technologies, we argue that 
with an increased average alliance portfolio (figure 10), in combination with a wave of firm entrance, the average number of different partners in a firms portfolio has grown. After all, these newly entering firms are deal-active and, given the complementarities of their new technologies, they have found new partners in the existing network. In sum, we can conclude that from the beginning of the nineties, firms have on average expanded their portfolio size and range. This leads us to confirm hypothesis 1, stating that the pharmaceutical R\&D network has expanded from the beginning of the nineties. From figure 9 and 10 it becomes clear however, that the average portfolio size of firms does not increase further after the beginning of the nineties and that the entrance of new firms into the network is also temporary. We can therefore conclude that although the R\&D network appears to keep growing over time, the overall structural network expansion is temporary. This finding is in line with similar findings from the steel industry where technological change leads to a temporary 'reshuffling' of relational behavior, causing temporary structural change in the network (Madhaven et al., 1998).

\section{Local link formation}

The second part of the analysis concerned a more detailed investigation of these new alliances that have been formed with new partners. We have argued that, depending on the new partner being active in 'the neighborhood' or being a 'distant' partner (or both) we expect to see different structures emerge. We start with the hypothesis of local link formation. If firms are oriented toward local link formation, we should witness an increasing clustering coefficient from the beginning of the nineties. 
Fig. 11 - Weighted overall clustering coefficient in the pharmaceutical R\&D network (full line) and of a random network (dashed line)

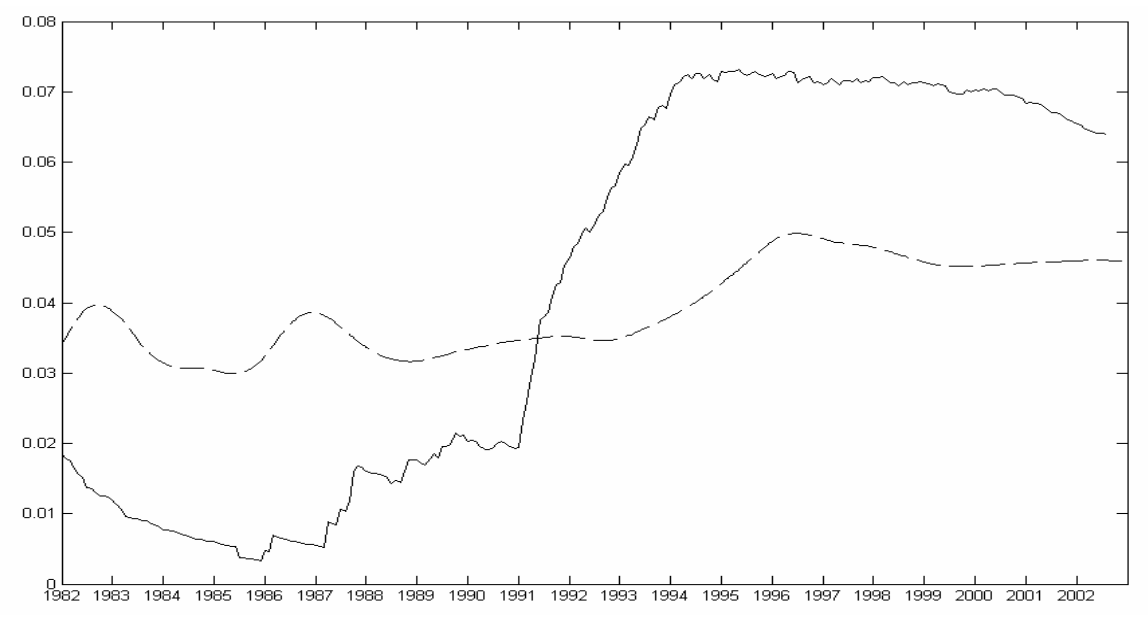

Figure 11 shows the weighted clustering coefficient from 1982 till 2002. Between 1991 till 1995 there is a sharp increase in the weighted clustering coefficient, while it starts to decrease slightly afterwards. This feature supports our hypothesis of an increased clustering from the beginning of the nineties, but in order to value the increase we have added the clustering coefficient of a simulated random network (Erdös-Renyi random network). A simulated random network is a much used tool in network analysis to indicate the meaning of a certain network value. The clustering coefficient of a random network with the same amount of actors and the same average degree (portfolio size) serves as a bench-mark to compare observed relational behavior with random relational behavior. When comparing the clustering coefficient of a random network in figure 11 with the observed clustering coefficient in our network, the impact of the genomics revolution on local clique formation becomes even more apparent. Before the beginning of the nineties, relational behavior of firms led to less clustering compared to random relational behavior, while after the beginning of the nineties there was more clique formation then was to be expected from random relational behavior. When considering the fact that a clustering coefficient is also used in the literature as a measure of local hierarchy, our relatively low clustering coefficient before the beginning of the nineties seems to support the results of Orsenigo et al. (2001). They argue that the pharmaceutical $R \& D$ network developed as a hierarchal branching process before the nineties. Finally we can confirm hypothesis 2 a through an increasing clustering coefficient after the genomics revolution, which indicates that firms have found new partners through local link formation. 


\section{Distant link formation}

Did the genomic revolution bring forth (new) firms that were able to bridge some of the 'hierarchal branches' of the pharmaceutical R\&D network? Given the hierarchal branching structure of the R\&D network together with the inherent characteristics of GPT we expect general purpose technology based firms, who have entered the network in the beginning of the nineties, to be able to bridge these hierarchies. If so, these bridges or distant linkages would function as shortcuts in the network and shorten the average path-length. Figure 12 reports the average path-length of our network and the average path-length of a simulated random network over time.

Fig. 12 - Average path-length of the pharmaceutical R\&D network (full line) and of a random network (dashed line)

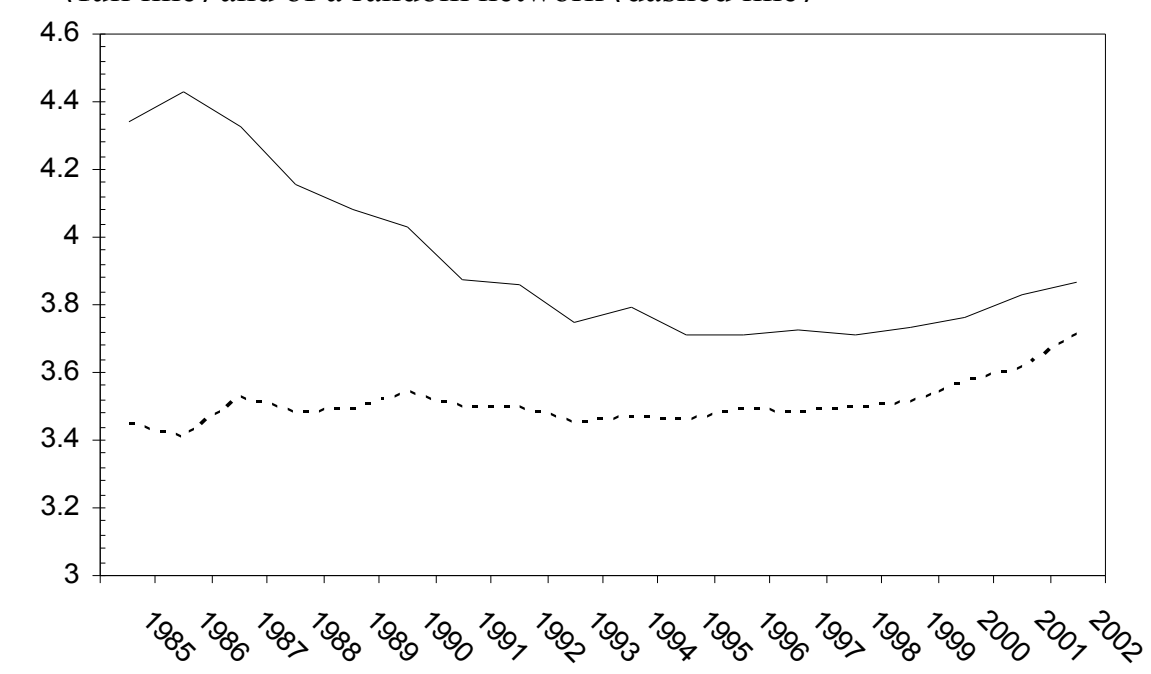

The average path-length declines from 1986, reaching its lowest point in 1995. This indicates that the reach between actors in the network has improved. After 1998 the average pathlength slightly starts to increase again. At first, it seems counterintuitive that the path-length shortens while the network grows. In our hypothesis we have argued that this shortening of the path-length has been triggered by the genomic revolution at the beginning of the nineties. Although the path-length indeed decreases, there is no indication that this decreasing trend was triggered by the genomic revolution, since the declining path-length clearly starts much earlier (from 1986). In order to value the decrease in path-length we have simulated a random network based on the exact same network size and connectivity at each time period $\tau$. The dashed line in figure 12 reports the average path-length of our simulated random network. In our random network an actor has to surpass 3.5 other actors on average to reach every other reachable actor in the network. As the network grows and becomes more connected this path- 
length remains stable but eventually will grow according to $\ln (\mathrm{n}) / \ln (\mathrm{k})(\mathrm{k}=$ average degree) for very large networks (see Watts, 1999). Starting from 1986, our empirical network and the random network slowly converge, which means that the reach between actors in the network improves despite a growing number of actors.

In sum, we can conclude that hypothesis $2 \mathrm{~b}$ predicting a falling average path-length from the beginning of the nineties cannot be confirmed. However, the fact that the path-length of our random network remains relatively stable indicates that there is non-random relational behavior causing an improvement in the reach between actors.

\section{Discussion \& Interpretation}

How can we interpret these results and what are its implications? To begin with we have clearly seen that the genomic revolution in the beginning of the nineties has caused structural network change. More specifically the network has expanded through an increasing average portfolio size and portfolio range, there has been a wave of firms entering and the technological focus of alliances has shifted from conventional medicinal chemistry to GPT. A second question was about the relational behavior that has caused this structural change, more specifically we studied whether firms find new partners through local linkages or whether these new partners are 'distant' partners. With this question we have extended the framework of Koka et al. (2006) on structural network change into more detail, but also we have combined the issue of partner choice in alliance networks with the study of small-world network structures. Our argument was that the relational behavior that causes a small-world structure in social networks is a combination of local link formation with relatively few distant linkages between different cliques. Together these two types of relational behavior image the combination of a regular network with a handful of random linkages, which defines a small-world according to Watts \& Strogatz (1998). Thirdly, we tried to find answers to what causes this specific relational behavior which in turn causes this specific structural change. There is basic agreement among network researchers that exogenous events, which increase uncertainty, cause an increase in alliance formation, but theories differ about the partner choice following from the decision to enter an alliance. Some network researchers (e.g. Powell, 1996; Burt 1991) argue that if firms enter into new alliances with new partners, they will use their network to find these new partners, which implies structural network change to be a local growth process. This argument leaves the empirical finding of small-world network structures unanswered however, because it fails to provide an explanation for the formation of distant linkages. We have argued that only a radical exogenous shock such as the genomic 
revolution can convince firms to leave their embedded path and form distant linkages with unknown partners. Following from this we hypothesized that after the genomic revolution, we would witness both local link formation and distant link formation, resembling a small-world. Although the evolution of the pharmaceutical R\&D network does show a decreasing average distance and increased clustering, which are indicators of a small-world, we find no evidence for our hypothesis that the genomic revolution has induced this structural change. One explanation for this result could be that general purpose based firms, whom we expected to form distant linkages between disease branches, do not perform these alliances. The fact that we witness the increase in clustering after the genomic revolution indicates that general purpose based firms find new partners through local linkages. This latter finding confirms the structure action dynamics in network evolution, where firms choose their alliance partner using their existing network. Our paper contributes an important detail to the structure action dynamics, namely that while the underlying technological base is stable (before the genomic revolution) new alliances are formed mainly with existing partners (given the low clustering coefficient) and that it requires an exogenous radical change for firms to engage into new alliances with new partners. But even when they do, they use their network to find these partners.

Although we find no evidence for our hypothesis that the genomic revolution has caused a decreasing average distance in the network, we do witness a temporary decreasing distance between 1986 and 1998, indicating that there are firms in the network who form distant linkages that shorten the distance between other organizations. The fact that these firms already performed this relational behavior before the genomic revolution might indicate that the network structure was not composed of relatively isolated branches as we assumed, but already consisted of some organizations that improved the overall reach in the network. Jackson (2006) proposes that not only distant linkages, but also highly connected actors called 'stars' or 'hubs' can cause a path-length to decrease. While it is beyond the scope of this paper to re-investigate the structure of the R\&D network before the beginning of the nineties, we did look for a simple indication to check Jackson's (2006) idea. Figure 13 plots the number of alliances for each 'type' of firm. 
Fig. 13 - Number of alliances per firm type

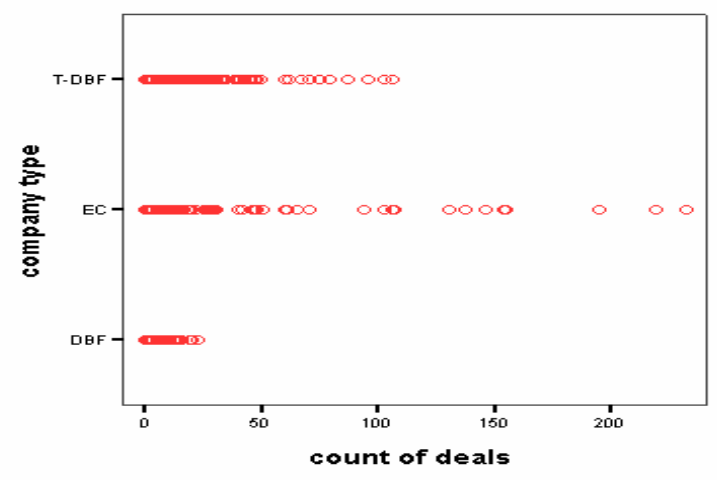

What becomes clear in this plot is that while there are quite some highly connected 'stars' amongst general purpose based firms (referred to as T-DBF), there are some pharmaceutical firms (EC) who are even more connected than general purpose based firms are. If these pharmaceutical 'stars' already connected different disease areas before the genomic revolution, then the addition of a few distant linkages by general purpose based firms would not cause a significant decrease in the average distance of the network. In other words, large pharmaceutical firms that are highly connected 'stars' already reduce the average distance in the network and general purpose based firms that bridge different disease areas contribute to the decreasing distance. Together these firms' alliances are responsible for the occurrence of a small-world. We feel these insights provide an interesting start for further research.

\section{Conclusion}

The genomic revolution in the beginning of the nineties has increased environmental uncertainty and munificence, and this has led to structural changes in the pharmaceutical $R \& D$ network. The network has expanded in terms of both number of firms and number of alliances. Since alliance activity outperformed the growth in the number of firms entering the network, we can conclude that the average number of deals and the average number of different partners has increased. On a firm level this means that new alliances have been formed with new partners, causing structural network change. The formation of new alliances with new partners can take to forms, implying different structural outcomes at the level of the network. First, firms can choose their new alliance partner through their existing network, which leads to local link formation and network clustering. Second, firms can form distant linkages with unknown partners. These linkages can potentially improve a firms' position in a network if it manages to connect previously unconnected parts of the network. Taken 
together, local linkages and distant linkages form a small-world network which has been a popular subject of recent network studies. While we argued that a radical exogenous shock would be required for firms to be forming distant linkages, we found no evidence of this alliance behavior. We found that firms, when confronted with radical technological change keep their existing alliances and form new alliances with partners of their partners. This result is consistent with previous studies on alliance strategies and network formation. Finally we provide an alternative explanation for the observed small-world characteristics through the identification of highly connected 'stars'. 


\section{References}

Anderson, M.L., and P. Tushman, (1986). 'Technological Discontinuities and Organizational Environments', Administrative Science Quarterly, 31(3) pp. 439-465.

Arora, A., and A. Gambardella, (1994). 'The changing technology of technological change: general and abstract knowledge and the division of innovative labour', Research policy, 23(5) pp. 523-532.

Albert, R., and A.L.Barabasi, (2002). 'Statistical mechanics of complex networks'. Reviews of Modern Physics 74(47) pp. 48-94.

Barley, S.R., (1986). 'Technology as an occasion for structuring: evidence from observations of CT scanners and the social order of radiology departments', Administrative Science Quarterly, 21(1) pp. 78-108.

Bourdieu, P., (1980). 'Le Capital Sociale: Notes Provisaires', Actes de la Recherche en Sciences Sociale 3 (2-3).

Borgatti, S., Everett, M., and L. Freeman, (1999). UCINET 5 for Windows: Software for Social Network Analysis. Analytic Technologies, Inc., Natick, MA.

Burckhardt, M.E., and D.J. Brass, (1990). 'Changing patterns or patterns of change: the effect of a change in technology on social network structure and power', Administrative Science Quarterly 35, pp.104-127.

Burt, R. S., (1992). Structural Holes: The Social Structure of Competition. Harvard University Press, Cambridge, MA

Coleman, J., (1990). 'Social Capital in the Creation of Human Capital', American Journal of Sociology 94.

Dess, G. G., and D. W. Beard, (1984). 'Dimensions of organizational task environments'. Administrative Science Quarterly, 29 pp. 52-73.

Dickson, P., and K.M. Weaver, (1997). 'Environmental determinants and individual level moderators of alliance use', Academy of Management Journal, 40 pp. 404-425.

Deroian, P., (1986). 'On the evolution of group and network structure: Structures within structure', Social Networks, 8 pp. 33-64.

Dorogovtsev, S.N., and J. F. F. Mendes, (2003). Evolution of Networks: From Biological Nets to the Internet and WWW. Oxford University Press, New York.

Drews, J., (2000) 'Drug discovery: a historical perspective', Science 287 pp. 1960-1964.

Gassmann, O., Reepmeyer, G., and M. von Zedtwitz, (2004). Leading Pharmaceutical Innovation, Trends and Drivers for Growth in the Pharmaceutical Industry. Springer, Berlin. Glasmeier A., (1991). The high-tech potential: Economic development in rural America. New 
Brunswick, NJ, Center for Urban Policy Research.

Goyal, S., Leij, M. J. van der, and J. L. Moraga-Gonzales, (2006). 'Economics: An Emerging Small World', Journal of Political Economy 114 (2) pp. 403-412.

Granovetter, M., (1983). 'The strength of weak ties: A network theory revisited', Sociological theory 1 pp. 201-233.

Granovetter, M., (1985). 'Economic action, social structure and Embeddedness', American Journal of Sociology 91 pp 481- 510.

Gulati, R., and M. Gargiulo, (1998). 'Where Do Interorganizational Networks Come From?', American Journal of Sociology 104 (5) pp. 439-93.

Gulati, R., (1998). ‘Alliances and networks', Strategic Management Journal 19 pp. 293-317.

Gulati, R., Nohria, N., and A. Zaheer, (2000). 'Strategic networks,' Strategic Management Journal 21 pp. 203-215.

Hannan, M. T., and J. Freeman, (1989). Organizational Ecology. Cambridge (MA) Harvard University Press.

Jackson, M.O., and B.W. Rogers, (2006) 'Meeting Strangers and Friends of Friends: How Random are Socially Generated Networks?’ American Economic Review, forthcoming.

Kaplan, S., Murray, F., and R. Henderson, (2003). 'Recognition and response to biotechnology by leading pharmaceuticals companies', Industrial \& Corporate Change 12 (4) pp. 203-233.

Kogut, B., (1991). 'Joint ventures and the option to expand and acquire', Management Science 37 pp. 19-33.

Kogut, B., (2000). 'The Network as Knowledge: Generative Rules and the Emergence of Structure', Strategic Management Journal 21 pp. 405-425.

Koka, R. B., Madhavan, R., and J. E. Prescott, (2006). 'The Evolution of Inter-firm Networks: environmental effects on patterns of network change', Academy of Management Review 31 (3) pp.721-737.

Krackhardt, D., (1992). 'The strength of strong ties: The importance of Philos in organizations'. In N. Nohria and R. Eccles (eds.), Networks and Organizations: Structure, Form and Action. Harvard Business School Press, Boston, MA.

Longman, R., (2000). 'Platform Technologies and the Collaboration Paradox', In VIVO. Pharmaventures (June).

Lytton, (1999). 'The New Structures of Platform Deals', Start-Up. Pharmaventures (Oktober). Madhavan, R., Koka, B. R., and J. E. Prescott, (1998). 'Networks in transition: How industry events (re)shape interfirm relationships', Strategic Management Journal 19 pp. 439-459.

Newman, M., (2001). 'The Structure of Scientific Collaboration Networks', Proceedings of the National Academy of Sciences 98 (01) pp. 4-9. 
Nightingale, P., (2000). 'Economies of Scale in Experimentation: knowledge and technology in pharmaceutical R\&D', Industrial \& Corporate Change 9 (2) pp. 315-359.

Nohria, N., and C. Garcia-Pont, (1991). 'Global strategic linkages and industry structure', Strategic Management Journal 12 pp. 105-124.

Nohria, N., (1992). Introduction: Is the network perspective a useful way of studying organizations? In N. Nohria \& R. Eccles (Eds.), Networks and organizations: Structure, form and action. Cambridge, MA, Harvard University Press.

Orsenigo, L. F., Pammolli, M., and M. Riccaboni, (2001). 'Technological Change and Network Dynamics: Lessons from the Pharmaceutical Industry', Research Policy 30 pp. 485508.

Pammolli, F., Orsenigo, L., and M. Riccaboni, (2001). Variety and Irreversibility in Scientific and Technological Systems: lessons from the Pharmaceutical Industry after the Molecular Biology Revolution. In U. Pagano, A. Nicita, eds. The Evolution of Economic Diversity. Routledge,

London.

Pfeffer, J., and G. R. Salancik, (1978). The External Control of Organizations: A Resource Dependence Perspective. Harper \& Row, New York.

Piore, M., and C. F. Sabel, (1984). The Second Industrial Divide. Basic Books, New York. Pisano, G., (1991). 'The Governance of Innovation. Vertical Integration and Collaborative Arrangements in the Biotechnology Industry', Research Policy 20 237-249.

Powell, W.W., Koput, K., and L. Smith-Doerr, (1996). 'Interorganizational Collaboration and the Locus of Innovation: Networks of Learning in Biotechnology', Administrative Science Quarterly 41(1) pp. 116-145.

Powell, W.W., Koput, K., White, D.R., and J. Owen-Smith, (2005). 'Network Dynamics and Field Evolution: The Growth of Interorganizational Collaboration in the Life Sciences' American Journal of Sociology 110(4) pp. 1132-1205.

Raub, W., and J. Weesie, (1990). 'Reputation and efficiency in social interactions: An example of network effects', American Journal of Sociology 96 pp. 626-654.

Pammolli, F., and M. Riccaboni, (2002). 'Technological Regimes and the Growth of Networks: An Empirical Analysis', Small Business Economics 19(3) pp 205-215.

Stuart, T. E., Hoang, H., and R.C. Hybels, (1999). 'Interorganizational endorsements and the performance of entrepreneurial ventures', Administrative Science Quarterly 44 pp. 315-349. Uppenbrink, J., and J. Mervis, (2000). 'An information Revolution' Science 287(5460) pp. 1951.

Uzzi, B., and J. Spiro, (2005). 'Collaboration and Creativity: The small-world Problem', American Journal of Sociology 111(2). 
Verspagen, B., Duysters, G.M., (2004). 'The small worlds of strategic technology alliances', Technovation, 24(7).

Watts, D., and S. Strogatz, (1998). 'Collective Dynamics of 'Small-World' Networks', Nature 393 pp. 440-442.

Wilhite, A., (2001). 'Bilateral Trade and 'Small-World' Networks', Computational Economics 18 49-64. 